\title{
Mitochondrial proteomics alterations in rat hearts following ischemia/reperfusion and diazoxide post-conditioning
}

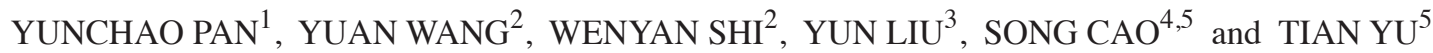 \\ ${ }^{1}$ Department of Anesthesiology, The Second Affiliated Hospital of Zunyi Medical University, Zunyi, \\ Guizhou 563000; ${ }^{2}$ Department of Anesthesiology, Affiliated Hospital of Zunyi Medical University; \\ ${ }^{3}$ Research Center for Medicine and Biology, Zunyi Medical University, Zunyi, Guizhou 563003; \\ ${ }^{4}$ Department of Pain Medicine, Affiliated Hospital of Zunyi Medical University, Zunyi, Guizhou 563000; \\ ${ }^{5}$ Guizhou Key Laboratory of Anesthesia and Organ Protection, Zunyi Medical University, Zunyi, Guizhou 563003, P.R. China
}

Received January 31, 2020; Accepted August 7, 2020

DOI: $10.3892 / \mathrm{mmr} .2020 .11800$

\begin{abstract}
Diazoxide post-conditioning (D-Post) has been shown to be effective in alleviating myocardial ischemia/reperfusion (I/R) injury; however, the specific mechanisms are not fully understood. In the present study, isolated rat hearts were subjected to I/R injury and D-Post. The mitochondria were extracted, and mitochondrial protein expression was detected in normal, I/R and D-Post hearts using two-dimensional electrophoresis and matrix-assisted laser desorption ionization-time of flight mass spectrometry. Differentially expressed proteins were then identified using comparative proteomics. In total, five differentially expressed proteins were identified between the I/R and D-Post hearts. Compared with the I/R hearts, the expression of NADH dehydrogenase (ubiquinone) flavoprotein 1 (NDUFV1), NADH-ubiquinone oxidoreductase $75 \mathrm{kDa}$ subunit (NDUFS1), 2-oxoglutarate dehydrogenase (OGDH) and ATP synthase $\alpha$ subunit (isoform CRA_b, gil149029482) was increased in D-Post hearts. In addition, the expression of another isoform of ATP synthase $\alpha$ subunit (isoform CRA_c, gil149029480) was decreased in the D-Post group compared with the I/R group. The expression profiles of NDUFV1, NDUFS1 and OGDH in the two groups were further validated via western blotting. The five differentially expressed proteins may be protective effectors in D-Post,
\end{abstract}

Correspondence to: Dr Song Cao, Department of Pain Medicine, Affiliated Hospital of Zunyi Medical University, 149 Dalian Street, Zunyi, Guizhou 563000, P.R. China

E-mail: caosong4321@163.com

Professor Tian Yu, Guizhou Key Laboratory of Anesthesia and Organ Protection, Zunyi Medical University, 6 West Xuefu Street, Zunyi, Guizhou 563003, P.R. China

E-mail: zunyiyutian@163.com

Key words: diazoxide, post-conditioning, ischemia/reperfusion injury, mitochondria, proteomics, heart as well as potential targets for the treatment of cardiac I/R injury.

\section{Introduction}

Ischemic heart diseases are amongst the leading causes of mortality worldwide (1-3), with a death rate of 46-324 deaths per 100,000 individuals/year (3). Early reperfusion is key for the effective recovery of the ischemic myocardium; however, reperfusion may also result in ischemia/reperfusion (I/R) injury (4). Under normal circumstances, mitochondria provide ATP for myocardial survival and contraction; however, they are also the site of myocardial oxidative stress and calcium overload during an I/R situation (4,5). Therefore, mitochondria are important factors (both preventative and causative) during myocardial I/R injury. Studies have shown that myocardial I/R injury and different types of conditioning can affect mitochondrial function (6-9). Furthermore, the expression levels of certain mitochondrial proteins are altered following myocardial I/R injury and ischemic preconditioning (10-12). Recently, it was reported that D-Post may protect Langendorff I/R hearts via the mitochondrial ATP-sensitive potassium channel (mitoK $\mathrm{K}_{\text {ATP }}$ ) and the hypoxia-inducible factor-1/hypoxia response element pathway (13).

Following the discovery in 1991 of mitoK $_{\text {ATP }}(14)$, which is located at the inner mitochondrial membrane, mitoK ${ }_{\text {ATP }}$ was demonstrated to be a trigger for the protective effects of ischemic pre-conditioning $(15,16)$. Pharmacological conditioning is currently being evaluated as an alternative method for the treatment of I/R injury (17). Similar myocardial protection can be obtained from drugs such as mitoK ${ }_{\text {ATP }}$ openers, including diazoxide $(15,18)$. A previous study observed that diazoxide post-conditioning (D-Post) is cardioprotective in I/R rat cardiomyocytes (12), which has been also reported by Penna et al (19). However, the mechanisms via which D-Post protects the myocardium against I/R injury have not been fully elucidated.

The aim of the present study was to analyze the differential expression of mitochondrial proteins in normal, I/R and D-Post rat hearts, which may aid the exploration of potential targets for the treatment of myocardial I/R injury. 


\section{Materials and methods}

Animals. A total of 65 male Sprague-Dawley rats (age, 16-20 weeks old, weight, 200-250 g) were purchased from the Center of Laboratory Animals of The Third Military Medical University (Chongqing, China). The rats were housed in cages with $12 \mathrm{~h}$ light/dark cycles, ad libitum access to food and water at a constant humidity $(50-60 \%)$ and temperature $\left(22 \pm 1^{\circ} \mathrm{C}\right)$. All animals received humane care in compliance with the Guide for the Care and Use of Laboratory Animals (20), and all experimental protocols were approved by the Animal Care and Use Committee of Zunyi Medical University.

Materials. SDS, ammonium persulfate, sucrose, acrylamide, methylene bis-acrylamide, mannitol, glycerol and glycine were purchased from Amresco, LLC. Diazoxide, Nycodenz ${ }^{\circledR}$, urea, thiourea, EDTA and tetramethylethylenediamine were obtained from Sigma-Aldrich (Merck KGaA). Protein quantification kits, immobilized $\mathrm{pH}$ gradient (IPG) strips, dithiothreitol (DTT), BIO-Lyte, CHAPS, agarose, bromophenol blue, $\beta$-mercaptoethanol, iodoacetamide and PVDF were acquired from Bio-Rad Laboratories, Inc. Anti-cytochrome $c$ oxidase subunit IV (COX IV; cat. no. ab14744), anti-2-oxoglutarate dehydrogenase (OGDH; cat. no. ab137773), anti-NADH dehydrogenase (ubiquinone) flavoprotein 1 (NDUFV1; cat. no. ab203208) and anti-NADH-ubiquinone oxidoreductase 75 kDa subunit (NDUFS1; cat. no. ab169540) antibodies were purchased from Abcam. All reagents were of analytical grade.

Perfusion protocol. Rats were intraperitoneally anesthetized using sodium pentobarbital $(40 \mathrm{mg} / \mathrm{kg}$ ) containing heparin $(250 \mathrm{U} / \mathrm{kg})$. When rats were in deep anesthesia and had lost the pinch reflex, rat hearts were rapidly excised and placed in cold $\mathrm{K}-\mathrm{H}$ solution $\left(2.50 \mathrm{mM} \mathrm{CaCl}{ }_{2}, 11.1 \mathrm{mM}\right.$ glucose, $4.75 \mathrm{mM} \mathrm{KCl}, 1.19 \mathrm{mM} \mathrm{KH}_{2} \mathrm{PO}_{4}, 118.00 \mathrm{mM} \mathrm{NaCl}, 1.19 \mathrm{mM}$ $\mathrm{MgCl}_{2} \bullet 6 \mathrm{H}_{2} \mathrm{O}$ and $24.80 \mathrm{mM} \mathrm{NaHCO}$, $\mathrm{pH}$ 7.40). Then, hearts were quickly removed and connected to a Langendorff perfusion system. Hearts were perfused with $37^{\circ} \mathrm{C} \mathrm{K}-\mathrm{H}$ buffer bubbled for $10 \mathrm{~min}$ before perfusion with $5 \% \mathrm{CO}_{2}$ and $95 \% \mathrm{O}_{2}$ at $5.8 \mathrm{kPa}$ for $20 \mathrm{~min}$ before equilibration. Exsanguination following heart removal resulted in rat mortality; death was confirmed from the loss of pinch reflexes and rigor mortis after the removal of heart. The control, I/R and D-Post hearts were perfused using the Langendorff apparatus as previously reported $(9,21,22)$, and the protocols are outlined in Fig. 1. I/R injury was induced by hypoxia in the ischemia session and subsequent reperfusion with oxygenated K-H solution.

A total of 30 rat hearts were randomly allocated to the Control, I/R and D-Post groups ( $\mathrm{n}=10 /$ group). For equilibration, all hearts were perfused using the Langendorff apparatus with K-H solution for $20 \mathrm{~min}$. Hearts in the Control group were continuously perfused with oxygenated K-H solution for $100 \mathrm{~min}$. After equilibration, the I/R and D-Post hearts were subjected to $40 \mathrm{~min}$ of ischemia; the I/R hearts were then reperfused with K-H solution for $60 \mathrm{~min}$, while the D-Post hearts were reperfused with diazoxide $(50 \mu \mathrm{M}$ in K-H solution) for $2 \mathrm{~min}$, and then with K-H solution for $58 \mathrm{~min}$. Cardiac functional parameters, including heart rate (HR), the maximum rate of the rise in intraventricular pressure $\left(\mathrm{dp} / \mathrm{dt}_{\max }\right)$, left ventricular developed pressure (LVDP) and left ventricular end-diastolic pressure (LVEDP) were recorded using the PowerLab system (ADInstruments) following equilibration (T1) and reperfusion (T2). At the end of equilibration, if premature systoles were $<2 / \mathrm{min}, \mathrm{HR}>250 \mathrm{bpm}$ and LVDP $>80 \mathrm{mmHg}$, the ventricular tissues were collected for further experimentation at the end of the reperfusion period.

Transmission electron microscopy (TEM). The cardiac tissues were evaluated via electron microscopy, which was conducted as previously reported $(9,23)$. Briefly, $1 \mathrm{~mm}^{3}$ of the left ventricle was fixed in $0.25 \%$ glutaraldehyde and $3 \%$ paraformaldehyde at room temperature for $2 \mathrm{~h}$. The tissues were then mounted with $1 \%$ osmic acid, dehydrated with acetone and embedded using ethoxyline $618\left(35^{\circ} \mathrm{C}\right.$ overnight, $45^{\circ} \mathrm{C}$ for $8 \mathrm{~h}, 60^{\circ} \mathrm{C}$ for $48 \mathrm{~h}$ ). The myocardial sections were cut (50 nm thickness), stained in uranyl acetate and lead citrate for 30 and $10 \mathrm{~min}$ at room temperature, respectively, and then photographed with a transmission electron microscope (HITACHI-H7500; Hitachi, Ltd.) and ultrastructural damage was evaluated using Flameng's scoring method (24) as previously reported (9).

Mitochondria extraction. The purity of the extracted mitochondria is important for the accuracy of mitochondrial proteomics analysis. As previously described (9), the ventricular tissues were cut and placed into ice-cold mitochondrion-separating medium (700 mM sucrose, $210 \mathrm{mM}$ mannitol, $1 \mathrm{mM}$ EDTA and $10 \mathrm{mM}$ Tris-HCl; pH 7.4), homogenized and centrifugated at $1,500 \mathrm{x} \mathrm{g}$ for $10 \mathrm{~min}$ at $4^{\circ} \mathrm{C}$. The supernatant was then centrifuged at $12,000 \mathrm{x} \mathrm{g}$ for $10 \mathrm{~min}$ at $4^{\circ} \mathrm{C}$ to harvest the crude mitochondria. Finally, Nycodenz density gradient medium was layered into an ultracentrifuge tube (Beckman Coulter, Inc.) with layers of the following concentrations: i) $34 \% 0.5 \mathrm{ml}$; ii) $30 \% 0.8 \mathrm{ml}$; iii) $25 \% 1.2 \mathrm{ml}$ (containing the crude mitochondrial solution); and iv) $20 \% 0.3 \mathrm{ml}$. The samples were then centrifugated at $100,000 \mathrm{xg}$ for $60 \mathrm{~min}$ at $4^{\circ} \mathrm{C}$ to obtain purified mitochondria. To confirm the mitochondrial purity, TEM was performed to evaluate the status of the mitochondria.

Two-dimensional electrophoresis (2-DE) of mitochondrial proteins. To obtain the mitochondrial proteins, mitochondrial pellets were dissolved in hydration loading buffer (7 M urea, $2 \mathrm{M}$ thiourea, $40 \mathrm{mM}$ Tris base, $4 \%$ CHAPS and $1 \%$ DTT), sonicated for $10 \mathrm{sec}$, and centrifugated at $12,000 \mathrm{x} \mathrm{g}$ at room temperature for $20 \mathrm{~min}$. 2-DE was performed as previously described $(9,25)$ according to the manufacturer's protocol (Bio-Rad Laboratories, Inc.), but with minor modifications.

A 24-cm IPG strip (pH 5-8) was rehydrated for $14 \mathrm{~h}$ with hydration buffer containing $500 \mu \mathrm{g}$ mitochondrial protein. Isoelectric focusing was carried out at $250 \mathrm{~V}$ for $1 \mathrm{~h}$ as follows: $1,000 \mathrm{~V}$ for $3 \mathrm{~h}, 4,000 \mathrm{~V}$ for $3 \mathrm{~h}$, and then with incremental increases of $10,000 \mathrm{~V}$ until reaching $80,000 \mathrm{~V} / \mathrm{h}$. IPG strips were placed in $8 \mathrm{ml}$ equilibration solution $(375 \mathrm{mM}$ Tris- $\mathrm{HCl}$, $6 \mathrm{M}$ urea, $20 \%$ glycerol, $2 \%$ SDS and $0.001 \%$ bromophenol blue) and protein separation was performed using a Bio-Rad system (Bio-Rad Laboratories, Inc.). The IPG strips were loaded onto a 12\% SDS-PAGE gel in running buffer $(192 \mathrm{mM}$ glycine, $25 \mathrm{mM}$ Tris and 0.1\% mM SDS; pH 8.3), and a constant current was applied for $16 \mathrm{~h}$. 


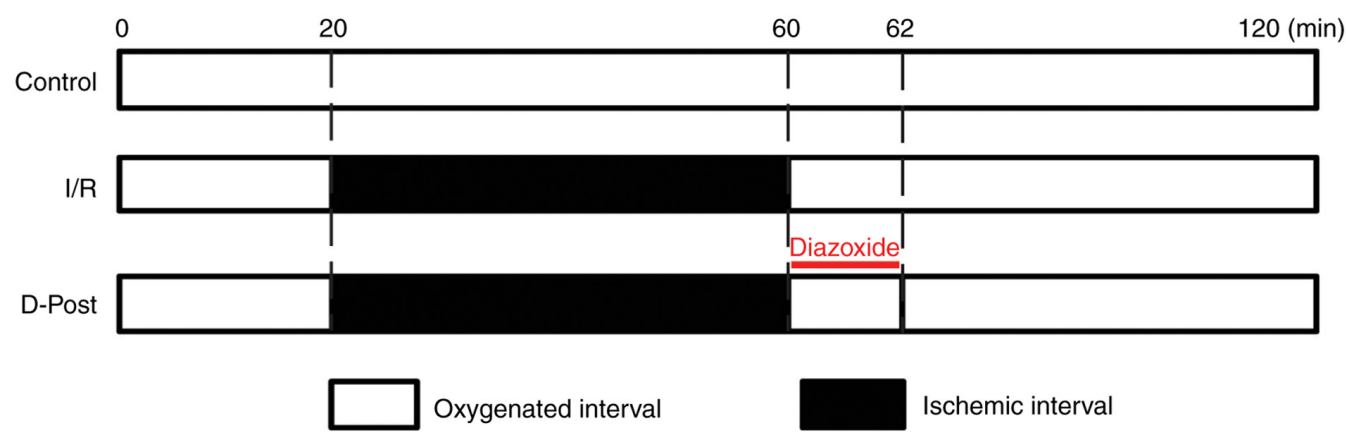

Figure 1. Perfusion protocol for cardiac I/R injury and D-Post. Hearts were categorized into three groups: i) Control; ii) I/R; and iii) D-Post. All hearts were equilibrated for $20 \mathrm{~min}$ before the different procedures were performed. Hearts in the Control group were continuously perfused for $100 \mathrm{~min}$. I/R hearts were subjected to ischemia for $40 \mathrm{~min}$ and reperfused for $60 \mathrm{~min}$. D-Post hearts received the same I/R treatment, and were then reperfused with K-H solution containing $50 \mu \mathrm{M}$ diazoxide for $2 \mathrm{~min}$ prior to reperfusion with $\mathrm{K}-\mathrm{H}$ solution for $58 \mathrm{~min}$. I/R, ischemia/reperfusion; D-Post, diazoxide post-conditioning.

Protein identification. Gels were stained with silver nitrate at room temperature for $30 \mathrm{~min}$ and digital images of protein dots on the gels were captured using a scanner (Seiko Epson Corporation). PDQuest 8.0 software (Bio-Rad Laboratories, Inc.) was used to identify differential spots between the Control, I/R and D-Post groups as previously described $(9,25)$, and these differential protein spots were excised from the gels and digested. The peptide mass fingerprint was obtained using matrix-assisted laser desorption ionization-time of flight mass spectrometry (MALDI-TOF MS) with a mass spectrometer (Ultraflex III; Bruker Corporation) and compared with that from the NCBInr protein database (http://www.matrixscience. com/help/seq_db_setup_nr.html) as reported previously (9). Peptides were extracted with $50 \mathrm{mM}$ NH4HCO3:ACN $(1: 1, \mathrm{v} / \mathrm{v})$. The peptide solution $(3 \mu \mathrm{l})$ was applied to a target disk to evaporate, and mixed with $0.1 \mu \mathrm{l}$ matrix solution ( $4 \mathrm{mg} / \mathrm{ml}$ in $70 \% \mathrm{ACN}$ and $30 \% 0.1 \% \mathrm{TFA}, \mathrm{v} / \mathrm{v}$ ), spectra was obtained with MALDI TOF/TOF mass. BioTools 3.0 (Bruker Corporation) and Mascot software (Matrix Science, Inc.) were the databases used to identify proteins via peptide mass fingerprinting. NCBInr was chosen as the sequence database. The names of the differentially expressed proteins were then confirmed.

Western blotting. Western blotting was conducted following standard procedures (26). The expression levels of $\beta$-actin, calnexin, GAPDH and COX IV in purified mitochondria were detected to confirm the purity of mitochondria used in the present study, and the expression levels of NDUFV1, NDUFS1 and OGDH were detected to compare with 2-DE results. Equal quantities of protein $(60 \mu \mathrm{g})$ from isolated mitochondria from Control, I/R and D-Post hearts were subjected to $12 \%$ SDS-PAGE, and transferred to PVDF membranes for immunoblotting. The membranes were blocked for $2 \mathrm{~h}$ at room temperature in TBS (20 mM Tris and $150 \mathrm{mM} \mathrm{NaCl}, \mathrm{pH} 8.0$ ), and then incubated at $4^{\circ} \mathrm{C}$ overnight with mouse anti- $\beta$-actin (cat. no. ab8226), rabbit anti-calnexin (cat. no. ab22595), mouse anti-GAPDH (cat. no. ab8245), mouse anti-COX IV (cat. no. ab33985), rabbit anti-NDUFV1 (cat. no. ab221998), rabbit anti-NDUFS1 (cat. no. ab169540) and rabbit anti-OGDH (cat. no. ab137773) primary antibodies (all 1:500 and purchased from Abcam). Then, membranes were subsequently incubated with HRP-conjugated anti-rabbit (cat. no. ab6721) or anti-mouse (cat. no. ab6728) secondary antibodies (1:2,000; Abcam). Protein expression was visualized via enhanced chemiluminescence (Cytiva) with a ChemiDoc MP system (Bio-Rad Laboratories, Inc. Image Lab software (version 5.2.1; Bio-Rad Laboratories, Inc.), NDUFV1, NDUFS1 and OGDH levels were normalized to that of COX IV.

Statistical analysis. All data are expressed as the mean \pm standard deviation. Comparisons of protein expression among groups were conducted with one-way ANOVA followed by Sidak's post hoc test for multiple comparisons. Comparisons at different time points in the same group, and comparisons at the same time point in 3 groups were conducted with two-way mixed ANOVA followed by Sidak's post hoc test for multiple comparisons. Comparisons of Flameng's score among different groups were conducted using Kruskal-Wallis test followed by Dunn's post hoc test for multiple comparisons. $\mathrm{P}<0.05$ was considered to indicate a statistically significant difference.

\section{Results}

D-Post improves cardiac function. D-Post effectively reversed I/R-induced hemodynamic dysfunction (Fig. 2). There was

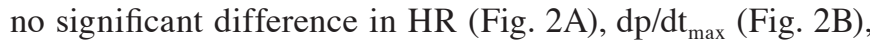
LVDP (Fig. 2C) or LVEDP (Fig. 2D) among the three groups at the end of T1. However, at the end of T2, the HR, dp/dt $\mathrm{max}_{\max }$ and LVDP of the Control and D-Post groups were significantly greater than those of the I/R group, whereas the LVEDP was significantly decreased in these two groups compared with I/R $(\mathrm{P}<0.05)$.

Electron microscopy. In the I/R group, the myocardial fibers were arranged in a disordered manner, the mitochondria were swollen, and the cristae were fractured and fuzzy (Fig. 3B). The myocardia of the D-Post group exhibited a more normal morphology; the myocardial fibers were arranged in an orderly manner, and fewer mitochondria were swollen (and to a lesser degree than those in the I/R group), but with an intact appearance (Fig. 3C). Quantification of mitochondrial damage was determined using Flameng's method (Fig. 3D). Flameng's score was considerably higher in the I/R group compared with the Control group (2.6 \pm 0.46 vs. $1.3 \pm 0.45)$, and subsequently decreased to $1.7 \pm 0.48$ in the D-Post group. 
A

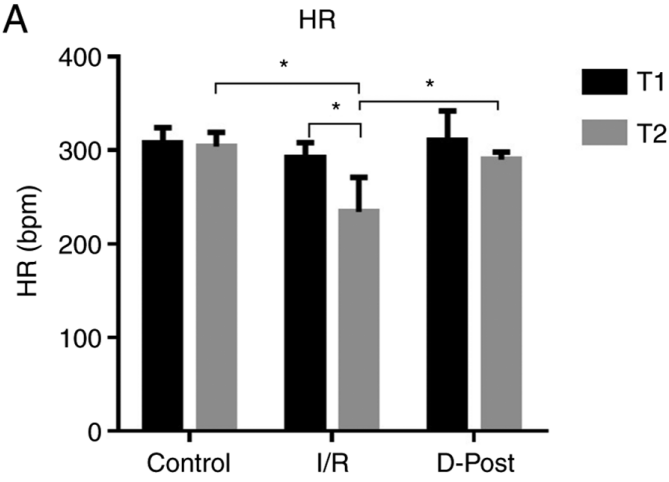

C

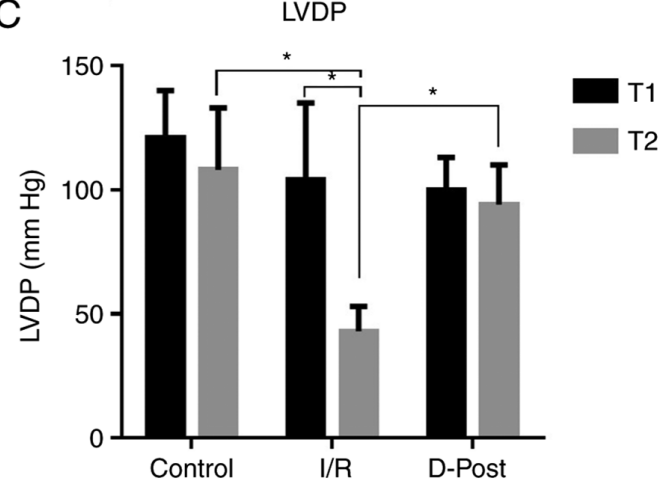

B

$\mathrm{dp} / \mathrm{dt} \mathrm{max}_{\max }$

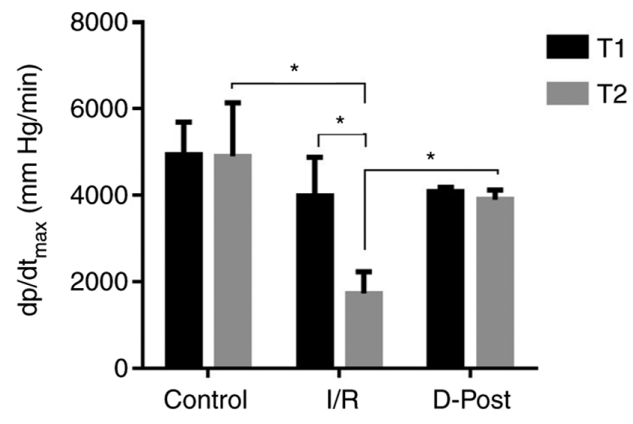

D

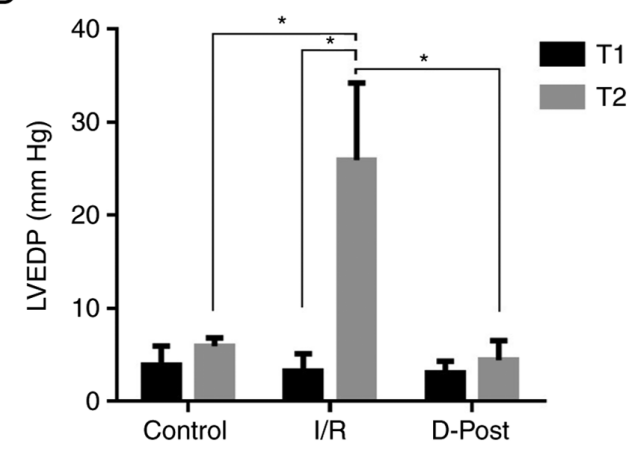

Figure 2. Comparison of hemodynamic parameters. D-Post effectively improves (A) HR, (B) dp/dt ${ }_{\max }$, (C) LVDP and (D) LVEDP after I/R injury. $\mathrm{n}=10 \mathrm{in}$ each group. Comparisons at different time points in the same group, and comparisons at the same time point in different groups were conducted using two-way ANOVA followed Sidak's post hoc test. "P<0.05. I/R, ischemia/reperfusion; D-Post, diazoxide post-conditioning; HR, heart rate; dp/dt max , maximum rate of the rise in intraventricular pressure; LVDP, left ventricular developed pressure; LVEDP, left ventricular end-diastolic pressure; T1, time point following equilibration; T2, time point following reperfusion.
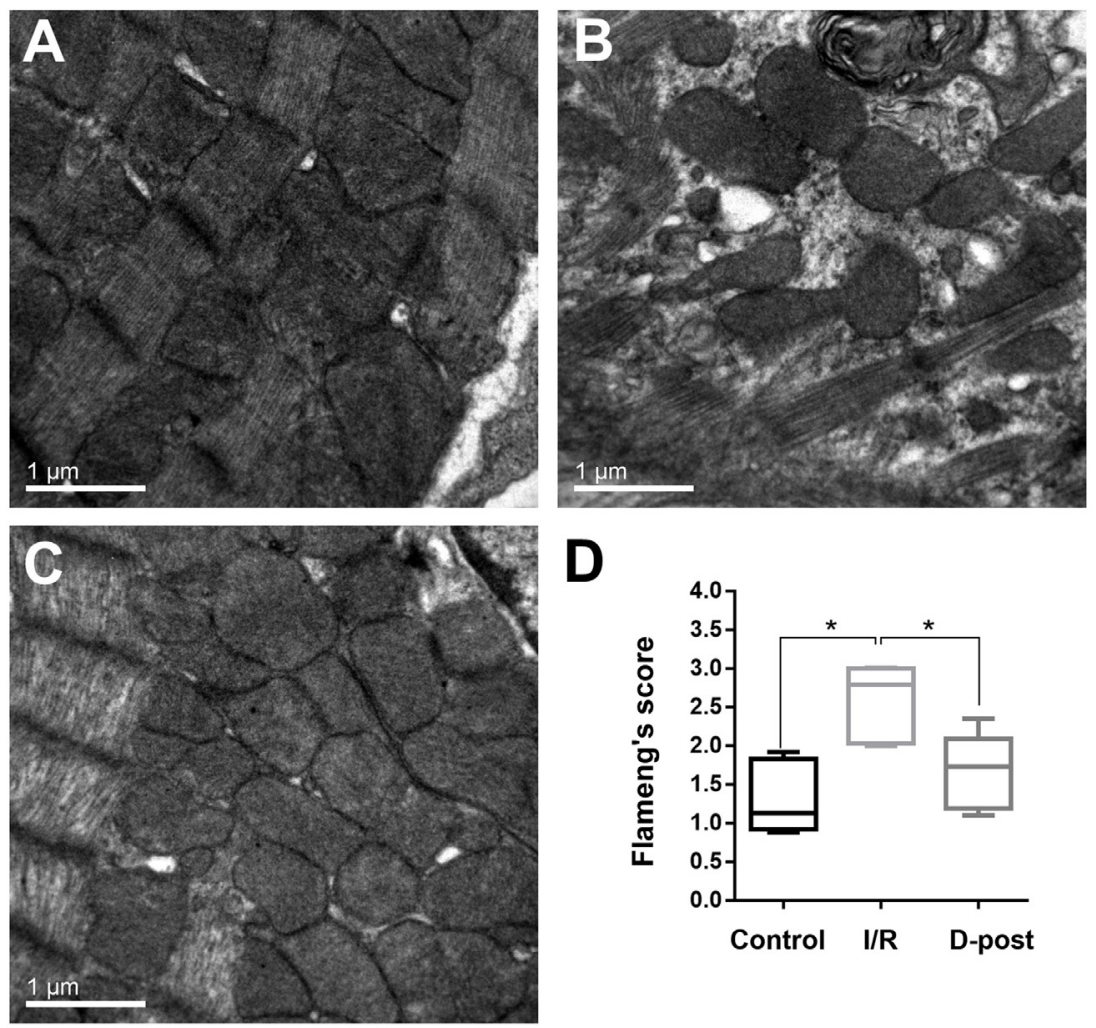

Figure 3. TEM analysis of myocardial damage. TEM images of Langendorff-perfused myocardia were analyzed following reperfusion in the (A) Control, (B) I/R and (C) D-Post groups. (D) Flameng's score of mitochondria from TEM images ( $\mathrm{n}=4 /$ group). Comparisons among different groups were conducted using Kruskal-Wallis test followed by the Dunn's post hoc test. " $\mathrm{P}<0.05$. TEM, transmission electron microscopy; I/R, ischemia/reperfusion; D-Post, diazoxide post-conditioning. 

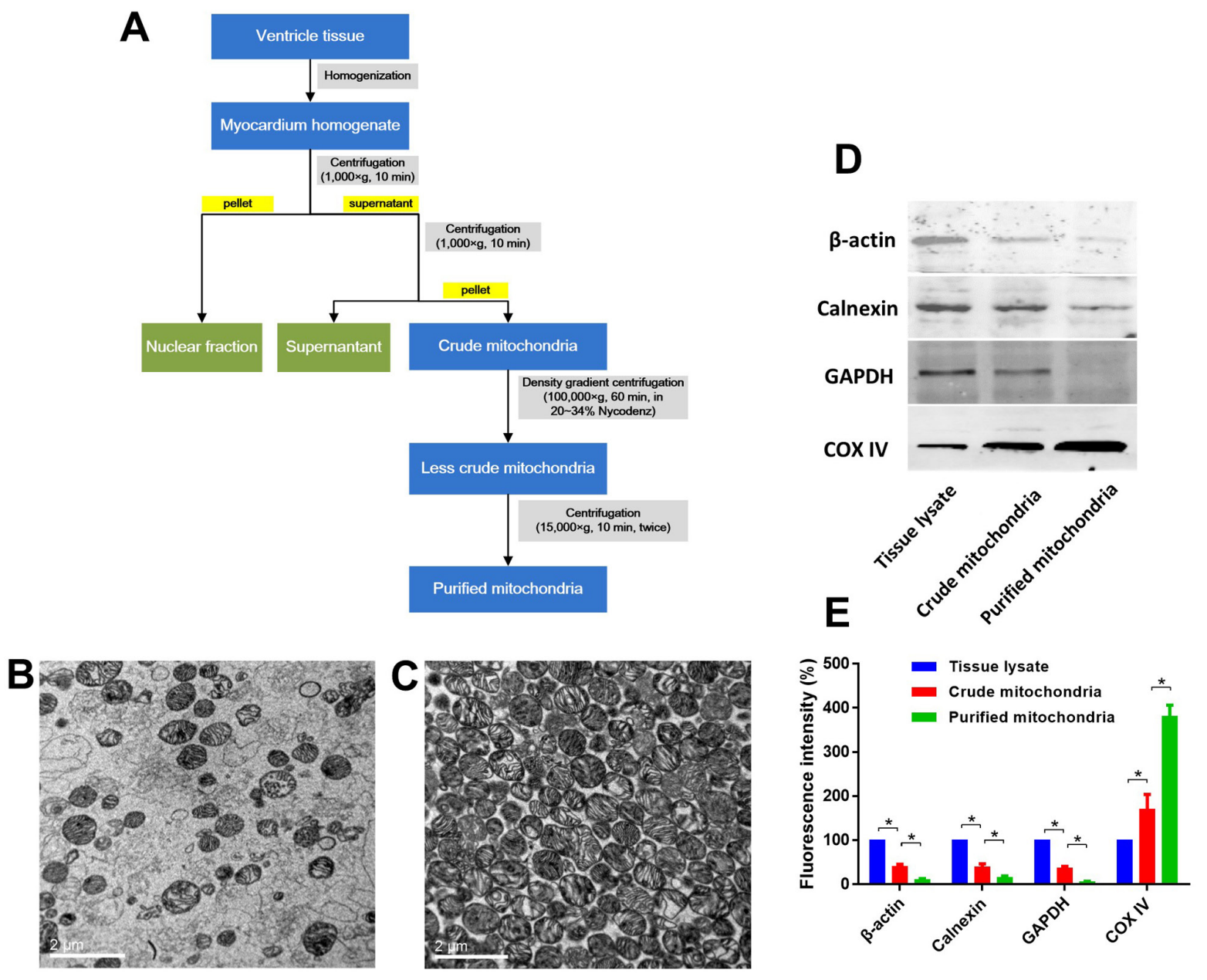

Figure 4. Flow chart of mitochondrial extraction and purification protocols. (A) Mitochondria were isolated using the Nycodenz density gradient centrifugation method. Representative transmission electron micrographs of (B) crude and (C) purified mitochondrial fractions from a heart in I/R group. Scale bar, $2 \mu \mathrm{m}$. (D) Evaluation of the expression of $\beta$-actin, calnexin, GAPDH and COX IV in the purified mitochondria via western blotting. (E) Expression levels of $\beta$-actin, calnexin and GAPDH in purified mitochondria were significantly decreased compared with in heart tissue and crude mitochondria, while the expression levels of COX IV in purified mitochondria were increased. $\mathrm{n}=4$ in each group. " $\mathrm{P}<0.05$. I/R, ischemia/reperfusion; D-Post, diazoxide post-conditioning; COX IV, cytochrome $c$ oxidase subunit IV.

Evaluation of mitochondrial purity. Mitochondria were extracted and purified as depicted in Fig. 4A. Mitochondrial purity was evaluated using TEM images and western blotting of $\beta$-actin, calnexin, GAPDH and COX IV, which indicated that high purity mitochondria were obtained (Fig. 4B-E). Representative TEM micrographs of the isolated mitochondria are displayed in Fig. 4. The mitochondria were intact with no swelling or rupturing, and the cristae were intact and organized (Fig. 4C). Few impurities were present within these fields of view (Fig. 4C). Western blotting data also indicated that the purified mitochondria exhibited high purity (high expression of COX IV, and low expression of $\beta$-actin, calnexin and GAPDH), as shown in Fig. 4D and E.

D-Post alters mitochondrial protein expression. The expression levels of mitochondrial proteins in the $\mathrm{I} / \mathrm{R}$ and $\mathrm{D}$-Post groups were compared following 2-DE. The gels were stained with silver nitrate and scanned, and representative images are presented in Fig. 5A (I/R) and 5B (D-Post). In total, 14 spots were identified with expression differences $>50 \%$ between the two groups (Fig. 5C).
Protein identification with MALDI-TOF MS. A total of 14 spots were isolated from the 2-DE gels of the I/R group, and subjected to MALDI-TOF MS. The peptide mass peaks were compared with those in the NCBInr protein database, revealing five differentially expressed proteins [NDUFV1, NDUFS1, OGDH, ATP synthase (isoform CRA_c, isoform CRA_b)]; descriptions of these five proteins are listed in Table I.

PPC alters the mitochondrial expression levels of NDUFVI, NDUFS1 and $O G D H$. To validate the data obtained from 2-DE and MALDI-TOF MS, three of the five differentially expressed proteins were selected and subjected to western blotting. The expression levels of NDUFV1, NDUFS1 and OGDH in the D-Post group were significantly upregulated compared with in the I/R group ( $\mathrm{P}<0.05$, Fig. 6), consistent with the 2-DE expression trends for these proteins.

\section{Discussion}

D-Post was previously revealed to effectively attenuate $I / R$ injury in primary adult rat cardiomyocytes (12). In the present ex vivo study, $\mathrm{D}$-Post alleviated $\mathrm{I} / \mathrm{R}$ injury in rat hearts. 
Table I. Information of differentially expressed proteins.

\begin{tabular}{|c|c|c|c|c|c|c|c|}
\hline Protein name & $\begin{array}{l}\text { Gene } \\
\text { name }\end{array}$ & Calculated PI & $\mathrm{SSP}^{\mathrm{a}}$ & NCBI GI no. & $\begin{array}{c}\text { AA } \\
\text { coverage }^{\mathrm{b}}, \%\end{array}$ & Fold-change $^{c}$ & P-value ${ }^{\mathrm{d}}$ \\
\hline $\begin{array}{l}\text { NADH dehydrogenase (ubiquinone) } \\
\text { flavoprotein } 1\end{array}$ & NDUFV1 & 5.89 & 1405 & 149061921 & 24 & $3.6 \pm 0.2$ & 0.002 \\
\hline 2-oxoglutarate dehydrogenase & OGDH & 6.30 & 4904 & 62945278 & 12 & $3.3 \pm 0.5$ & 0.005 \\
\hline $\begin{array}{l}\text { NADH-ubiquinone oxidoreductase } \\
75 \mathrm{kDa} \text { subunit }\end{array}$ & NDUFS1 & 5.74 & 5604 & 149046009 & 24 & $2.6 \pm 0.5$ & 0.008 \\
\hline $\begin{array}{l}\text { ATP synthase, } \mathrm{H}+\text { transporting, } \\
\text { mitochondrial } \mathrm{F} 1 \text { complex, } \alpha \text { subunit, } \\
\text { isoform } 1 \text {, isoform CRA_c }\end{array}$ & ATP5F1A & 9.50 & 7704 & 149029482 & 25 & $2.5 \pm 0.8$ & 0.003 \\
\hline $\begin{array}{l}\text { ATP synthase, } \mathrm{H}+\text { transporting, } \\
\text { mitochondrial } \mathrm{F} 1 \text { complex, } \alpha \text { subunit, } \\
\text { isoform } 1 \text {, isoform CRA_b }\end{array}$ & ATP5F1A & 6.92 & 2107 & 149029480 & 38 & $-2.4 \pm 0.7$ & 0.001 \\
\hline
\end{tabular}

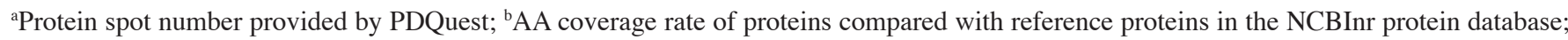
${ }^{\mathrm{c}}$ Fold-change in D-Post group compared with $\mathrm{I} / \mathrm{R}, \mathrm{n}=4$; negative values indicate downregulation in D-Post group; ${ }^{\mathrm{d}} \mathrm{T}$-test of expression (in parts per million) between I/R and D-Post groups. D-Post, diazoxide post-conditioning; GI, GenInfo Identifier; I/R, ischemia/reperfusion; NCBI, National Center for Biotechnology Information; PI, isoelectric point; SSP, significant spot.

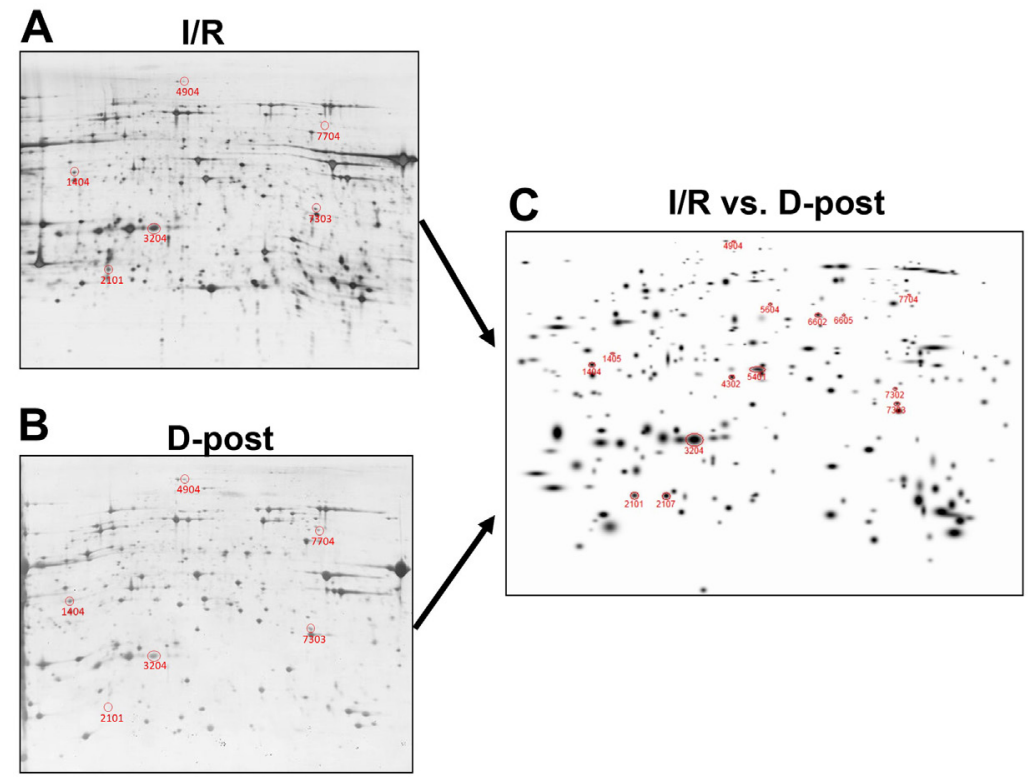

Figure 5. Proteomics comparison of differentially expressed proteins in rat myocardial mitochondria. Mitochondrial proteins were separated in (A) I/R and (B) D-Post fractions using linear immobilized $\mathrm{pH}$ gradient strips and SDS gels, which were subsequently stained with silver nitrate. (C) Differentially expressed protein spots (expression level difference $>2$-fold) with differential part per million values were annotated with numbers in an image synthesized by PDQuest software. I/R, ischemia/reperfusion; D-Post, diazoxide post-conditioning.

Studies have shown that mitoK $\mathrm{K}_{\text {ATP }}$ opening may be the trigger point and end-effector of the myocardial protective effects of certain drugs (27-29). Therefore, comparative mitochondrial proteomics analyses were used to detect potential effectors responsible for the protective effects of D-Post. As a result, five differentially expressed proteins between I/R and D-Post hearts were identified, all of which are associated with the mitochondrial respiratory chain or energy metabolism, and may therefore be potential myocardial protective effectors for $\mathrm{I} / \mathrm{R}$.
In the present study, the expression of NDUFV1 and NDUFS1 was increased following D-Post in I/R hearts. These $\mathrm{NADH}$ dehydrogenase subunits constitute the catalytic core of complex I $(30,31)$; the overexpression of NDUFV1 and NDUFS1 may therefore enhance the function of complex I and restore energy production in $\mathrm{I} / \mathrm{R}$, in which a lack of oxygen retards oxidation reactions and energy generation.

OGDH is one of the components of the ketoglutarate dehydrogenase complex, which is a key regulatory point in the tricarboxylic acid cycle, and catalyzes the oxidative 

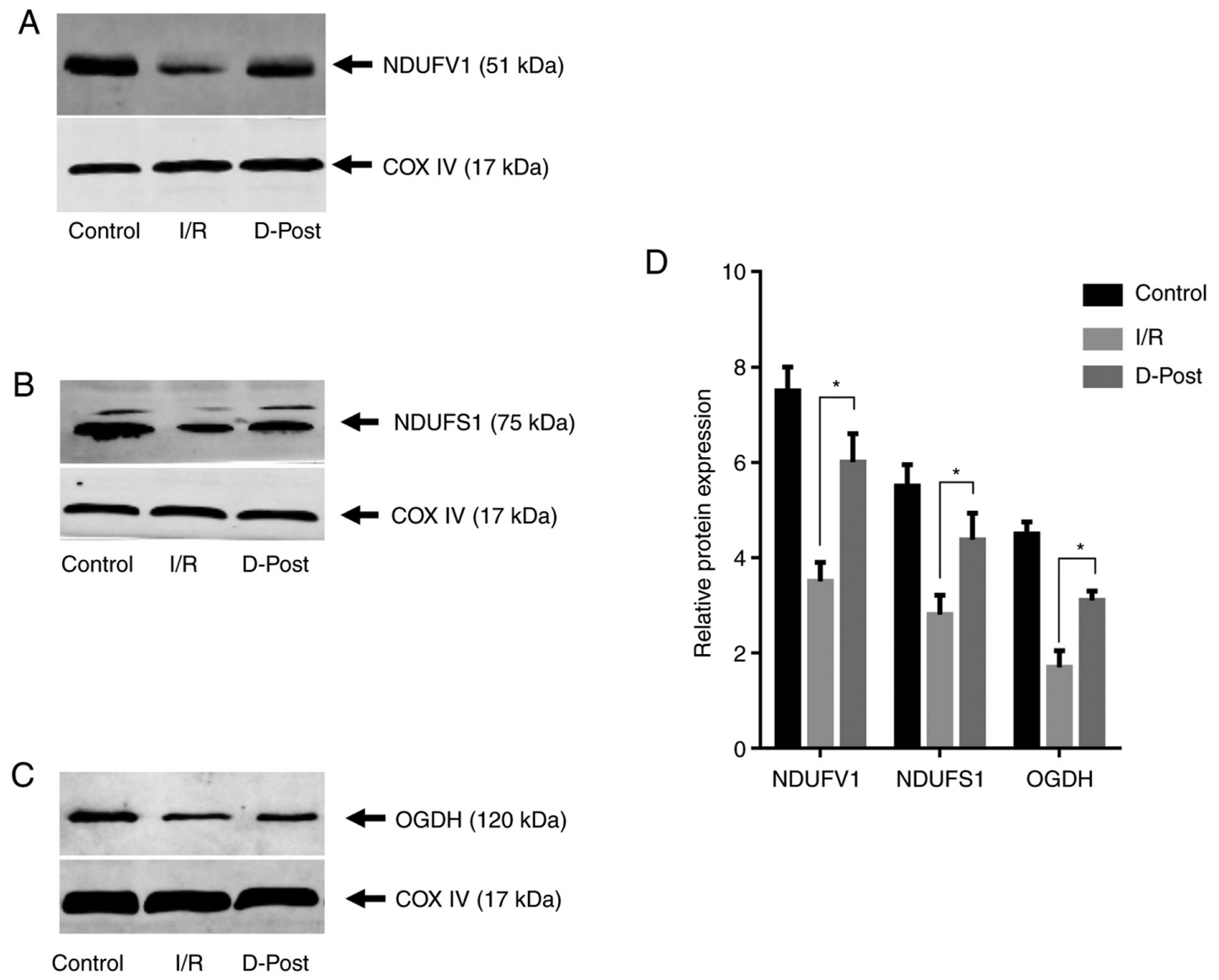

Figure 6. Expression levels of NDUFV1, NDUFS1 and OGDH in normal, I/R and D-Post hearts. (A) NDUFV1, (B) NDUFS1 and (C) OGDH expression as determined via western blotting. (D) Compared with the I/R group, expression of each protein was upregulated in the D-Post group, which was consistent with the expression trends observed with two-dimensional electrophoresis. $n=3$ in each group. * P $<0.05$. I/R, ischemia/reperfusion; D-Post, diazoxide post-conditioning; COX IV, cytochrome $c$ oxidase subunit IV; NDUFV1, NADH dehydrogenase (ubiquinone) flavoprotein 1; NDUFS1, NADH-ubiquinone oxidoreductase $75 \mathrm{kDa}$ subunit; OGDH, 2-oxoglutarate dehydrogenase.

decarboxylation of $\alpha$-ketoglutarate to succinyl-CoA, NADH and $\mathrm{CO}_{2}$ (32). In addition, $\mathrm{OGDH}$ also regulates mitochondrial redox potential (NADH/NAD+), and has been reported to be a significant source of reactive oxygen species (ROS) during mitochondrial succinate metabolism in the porcine heart (33). OGDH expression is reportedly decreased in the I/R heart $(10,34)$, which may influence ROS and energy production in the myocardium. In the present study, 2-DE and western blotting revealed an increase in OGDH expression following D-Post, and this recovery in expression may restore ROS and energy homeostasis in the I/R heart, thus decreasing damage to the ischemic myocardium.

The expression of ATP synthase subunit $\alpha$ (ATPA) was found to be altered in I/R hearts subjected to D-Post. Of note, two isoforms of ATPA were revealed to be differentially expressed; the CRA_c isoform was upregulated in D-Post hearts, while the CRA_b isoform was downregulated. As there are no specific antibodies that differentiate between these two isoforms, their protein expression levels cannot be separately detected by western blotting. I/R has been reported to promote the inhibition of ATP synthase, and subsequently decrease ATP production $(35,36)$. In addition, ATP synthase contributes to the effects of mitoK $\mathrm{ATP}_{\mathrm{T}}$ opening in I/R hearts $(37,38)$. D-Post increases ATP synthase activity, which may be a direct reason for its cardioprotective effects (38). However, whether the effects of D-Post are specifically associated with ATPA is yet to be investigated.

The present proteomics analysis of mitochondrial proteins between $I / R$ and D-Post rat hearts is a practical way to highlight potential D-Post effector proteins. A total of five differentially expressed proteins were identified using 2-DE and MALDI-TOF MS (and validated via western blotting), and each warrants further investigation. Proteomic changes between normal Langendorff and I/R hearts have been investigated previously and 4 differentially expressed proteins (ATPA, isoform 2 of cytochrome $c 1$, electron-transferring flavoprotein and NDUFS2) have been identified with the same protocol (25). Therefore, the differentially expressed proteins between the Control and I/R groups were not investigated in the present study.

There were limitations to the present study: i) The function of these differentially expressed proteins in I/R and D-Post tissues were not studied further (for example, it is not clear whether interventions in the expression of these proteins may affect I/R injury in the myocardium); ii) the use of narrow-range $\mathrm{pH}$ IPG strips ( $\mathrm{pH} 5-8$ ) may result in other potential proteins not being detected, which may be the reason that only five differentially expressed proteins were identified between the I/R and D-Post hearts; iii) for the evaluation of the protective effects of D-Post, triphenyltetrazolium chloride staining of 
Langendorff hearts would be a more convincing indicator and should be used to measure myocardial infarct area; and iv) as mentioned above, the lack of a normal control group for the 2-DE experiments is a limitation of the present study.

\section{Acknowledgements}

Not applicable.

\section{Funding}

The present study was supported by grants from The Special Scientific Research Fund for Public Welfare, Ministry of Health of China (grant no. 200802173) and the National Natural Science Foundation of China (grant no. 8176020318).

\section{Availability of data and materials}

The datasets used and/or analyzed during the current study are available from the corresponding author on reasonable request.

\section{Authors' contributions}

YP and YW confirm the authenticity of all the raw data. YP, YW, WS and SC performed the experiments, wrote the manuscript and prepared the figures. SC, YL and TY conceived the study, and provided the reagents and materials. All authors reviewed the data and drafts of the manuscript. All authors read and approved the final manuscript.

\section{Ethics approval and consent to participate}

All animals received humane care in compliance with the Guide for the Care and Use of Laboratory Animals in China, and all experimental protocols were approved by the Animal Care and Use Committee of Zunyi Medical University (Zunyi, China).

\section{Patient consent for publication}

Not applicable.

\section{Competing interests}

The authors declare that they have no competing interests.

\section{References}

1. Paiva $\mathrm{S}$ and Agbulut O: MiRroring the multiple potentials of MicroRNAs in acute myocardial infarction. Front Cardiovasc Med 4: 73, 2017.

2. Kaski JC, Crea F, Gersh BJ and Camici PG: Reappraisal of ischemic heart disease. Circulation 138: 1463-1480, 2018.

3. Nowbar AN, Gitto M, Howard JP, Francis DP and Al-Lamee R: Mortality from ischemic heart disease. Circ Cardiovasc Qual Outcomes 12: e005375, 2019.

4. Kuznetsov AV, Javadov S, Margreiter R, Grimm M, Hagenbuchner $\mathbf{J}$ and Ausserlechner MJ: The role of mitochondria in the mechanisms of cardiac ischemia-reperfusion injury. Antioxidants (Basel) 8: 454, 2019.

5. Lesnefsky EJ, Chen Q, Tandler B and Hoppel CL: Mitochondrial dysfunction and myocardial ischemia-reperfusion: Implications for novel therapies. Annu Rev Pharmacol Toxicol 57: 535-565, 2017.
6. Meng W, Xu Y, Li D, Zhu E, Deng L, Liu Z, Zhang G and Liu H: Ozone protects rat heart against ischemia-reperfusion injury: A role for oxidative preconditioning in attenuating mitochondrial injury. Biomed Pharmacother 88: 1090-1097, 2017.

7. Yang L, Xie P, Wu J, Yu J, Yu T, Wang H, Wang J, Xia Z and Zheng H: Sevoflurane postconditioning improves myocardial mitochondrial respiratory function and reduces myocardial ischemia-reperfusion injury by up-regulating HIF-1. Am J Transl Res 8: 4415-4424, 2016

8. Pagliaro P, Femmino S, Popara J and Penna C: Mitochondria in cardiac postconditioning. Front Physiol 9: 287, 2018.

9. Cao S, Liu Y, Wang H, Mao X, Chen J, Liu J, Xia Z, Zhang L, Liu $\mathrm{X}$ and $\mathrm{Yu} \mathrm{T}$ : Ischemic postconditioning influences electron transport chain protein turnover in Langendorff-perfused rat hearts. PeerJ 4: e1706, 2016.

10. Kim N, Lee Y, Kim H, Joo H, Youm JB, Park WS, Warda M, Cuong DV and Han J: Potential biomarkers for ischemic heart damage identified in mitochondrial proteins by comparative proteomics. Proteomics 6: 1237-1249, 2006.

11. Chen Q, Younus MS, Thompson J, Hu Y, Hollander JM and Lesnefsky EJ: Intermediary metabolism and fatty acid oxidation: Novel targets of electron transport chain driven injury during ischemia and reperfusion. Am J Physiol Heart Circ Physiol 314: H787-H795, 2018.

12. Cao S, Liu Y, Sun W, Zhao L, Zhang L, Liu X and Yu T: Genome-wide expression profiling of anoxia/reoxygenation in rat cardiomyocytes uncovers the role of MitoKATP in energy homeostasis. Oxid Med Cell Longev 2015: 756576, 2015.

13. Li J,Zhou W, Chen W, Wang H, Zhang Y and Yu T: Mechanism of the hypoxia inducible factor $1 /$ hypoxic response element pathway in rat myocardial ischemia/diazoxide post-conditioning. Mol Med Rep 21: 1527-1536, 2020.

14. Inoue I, Nagase $\mathrm{H}$, Kishi $\mathrm{K}$ and Higuti T: ATP-sensitive $\mathrm{K}^{+}$ channel in the mitochondrial inner membrane. Nature 352 : 244-247, 1991.

15. Garlid KD, Paucek P, Yarov-Yarovoy V, Murray HN, Darbenzio RB, D'Alonzo AJ, Lodge NJ, Smith MA and Grover GJ: Cardioprotective effect of diazoxide and its interaction with mitochondrial ATP-sensitive $\mathrm{K}^{+}$channels Possible mechanism of cardioprotection. Circ Res 81: 1072-1082, 1997.

16. Liu Y, Sato T, O'Rourke B and Marban E: Mitochondrial ATP-dependent potassium channels: Novel effectors of cardioprotection? Circulation 97: 2463-2469, 1998.

17. Caricati-Neto A, Errante PR and Menezes-Rodrigues FS: Recent advances in pharmacological and Non-pharmacological strategies of cardioprotection. Int J Mol Sci 20: 4002, 2019.

18. Garlid KD, Paucek P, Yarov-Yarovoy V, Sun X and Schindler PA: The mitochondrial KATP channel as a receptor for potassium channel openers. J Biol Chem 271: 8796-8799, 1996.

19. Penna C, Perrelli MG, Tullio F, Angotti C, Camporeale A, Poli V and Pagliaro P: Diazoxide postconditioning induces mitochondrial protein S-nitrosylation and a redox-sensitive mitochondrial phosphorylation/translocation of RISK elements: No role for SAFE. Basic Res Cardiol 108: 371, 2013.

20. National Research Council (US) Committee for the Update of the Guide for the Care and Use of Laboratory Animals: Guide for the Care and Use of Laboratory Animals. 8th edition. National Academies Press, Washington (DC), 2011. The National Academies Collection: Reports funded by National.

21. Yu T, Fu XY, Liu XK and Yu ZH: Protective effects of pinacidil hyperpolarizing cardioplegia on myocardial ischemia reperfusion injury by mitochondrial KATP channels. Chin Med J (Engl) 124: 4205-4210, 2011.

22. Yu T, Yu Z, Liu X, Yang S and Ye Y: Myocardial protection with pinacidil induced hyperpolarized arrest during cardiopulmonary bypass. Chin Med J (Engl) 114: 1245-1248, 2001.

23. Yang $\mathrm{L}$ and $\mathrm{Yu} \mathrm{T}$ : Prolonged donor heart preservation with pinacidil: The role of mitochondria and the mitochondrial adenosine triphosphate-sensitive potassium channel. J Thorac Cardiovasc Surg 139: 1057-1063, 2010.

24. Flameng W, Borgers M, Daenen W and Stalpaert G: Ultrastructural and cytochemical correlates of myocardial protection by cardiac hypothermia in man. J Thorac Cardiovasc Surg 79: 413-424, 1980.

25. Wei Y,Li K, Wang H, et al: Potential biomarkers for myocardial ischemia-reperfusion injury and pinacidil post-conditioning identified with mitochondrial proteomics in rats. Int J Clin Exp Med 12: 5060-5068, 2019. 
26. Cao S, Xiao Z, Sun M and Li Y: D-serine in the midbrain periaqueductal gray contributes to morphine tolerance in rats. Mol Pain 12: 1744806916646786, 2016.

27. Testai L, Rapposelli S, Martelli A, Breschi MC and Calderone V: Mitochondrial potassium channels as pharmacological target for cardioprotective drugs. Med Res Rev 35: 520-553, 2015.

28. Mironova GD, Rozova EV, Belosludtseva NV and Man'kovskaya IN: Dynamic restructuring of the myocardial mitochondria in response to uridine modulation of the activity of mitochondrial ATP-Dependent potassium channel under conditions of acute hypoxic hypoxia. Bull Exp Biol Med 166: 806-810, 2019.

29. Henn MC, Janjua MB, Kanter EM, Makepeace CM, Schuessler RB, Nichols CG and Lawton JS: Adenosine triphosphate-sensitive potassium channel kir subunits implicated in cardioprotection by diazoxide. J Am Heart Assoc 4: e002016, 2015.

30. Bjorkman K, Sofou K, Darin N, Holme E, Kollberg G, Asin-Cayuela J, Holmberg Dahle KM, Oldfors A, Moslemi AR and Tulinius M: Broad phenotypic variability in patients with complex I deficiency due to mutations in NDUFS1 and NDUFV1. Mitochondrion 21: 33-40, 2015.

31. Benit P, Chretien D, Kadhom N, de Lonlay-Debeney P, Cormier-Daire V, Cabral A, Peudenier S, Rustin P, Munnich A and Rötig A: Large-scale deletion and point mutations of the nuclear NDUFV1 and NDUFS1 genes in mitochondrial complex I deficiency. Am J Hum Genet 68: 1344-1352, 2001.

32. Veys K, Alvarado-Diaz A and De Bock K: Measuring glycolytic and mitochondrial fluxes in endothelial cells using radioactive tracers. Methods Mol Biol 1862: 121-136, 2019.
33. Mailloux RJ, Gardiner D and O'Brien M: 2-Oxoglutarate dehydrogenase is a more significant source of $\mathrm{O}_{2} \cdot-/ \mathrm{H}_{2} \mathrm{O}_{2}$ than pyruvate dehydrogenase in cardiac and liver tissue. Free Radic Biol Med 97: 501-512, 2016.

34. Kumar V, Kleffmann T, Hampton MB, Cannell MB and Winterbourn CC: Redox proteomics of thiol proteins in mouse heart during ischemia/reperfusion using ICAT reagents and mass spectrometry. Free Radic Biol Med 58: 109-117, 2013.

35. Granger DN and Kvietys PR: Reperfusion injury and reactive oxygen species: The evolution of a concept. Redox Biol 6: 524-551, 2015.

36. Bagheri F, Khori V, Alizadeh AM, Khalighfard S, Khodayari S and Khodayari $\mathrm{H}$ : Reactive oxygen species-mediated cardiac-reperfusion injury: Mechanisms and therapies. Life Sci 165: 43-55, 2016.

37. Ala-Rami A, Ylitalo KV and Hassinen IE: Ischaemic preconditioning and a mitochondrial KATP channel opener both produce cardioprotection accompanied by F1F0-ATPase inhibition in early ischaemia. Basic Res Cardiol 98: 250-258, 2003.

38. Jasova M, Kancirova I, Murarikova M, Farkašová V, Waczulíková I, Ravingerová T, Ziegelhöffer A and Ferko M: Stimulation of mitochondrial ATP synthase activity-a new diazoxide-mediated mechanism of cardioprotection. Physiol Res 65 (Suppl 1): S119-S127, 2016.

(i) (9) This work is licensed under a Creative Commons (c) $\mathrm{EY}$ No No Attribution-NonCommercial-NoDerivatives 4.0 International (CC BY-NC-ND 4.0) License. 